\title{
Um modelo de maturidade colaborativa: proposta inicial e validaçao de conteúdo
}

\author{
Humberto Falcão Martins y Ricardo Ribas da Costa \\ Instituto Publix
}

\section{Resumo}

Este trabalho busca: a) desenvolver um conceito de maturidade colaborativa, a partir da sistematização ajustada do conceito de governança colaborativa elaborada por Emerson y Nabatchi (2015a), aperfeiçoando o modelo anteriormente elaborado por Costa (2016), e de elementos e questões usualmente presentes em modelos consagrados de maturidade; b) operacionalizar o conceito, por meio da construção de um instrumento de mensuração da maturidade colaborativa; e c) testar a validade de conteúdo da operacionalização a partir da validade semântica do instrumento vis-à-vis o modelo de Emerson. O texto está divido em cinco partes. Uma introdução trata de apresentar as questões metodológicas que pertinem à construção de modelos. A parte dois trata de governança colaborativa e apresenta a sistematização elaborada por Emerson et al. (2012). A parte três trata de maturidade e apresenta questões metodológicas relacionadas à construção de modelos de maturidade. A parte quatro trata da construção do modelo de maturidade colaborativa e sua validação de conteúdo. A última parte elabora considerações sobre limitações e prosseguimentos.

Palavras-chave: Maturidade colaborativa, governança, validação de conteúdo.

A collaborative maturity model: initial proposal and validation of content

\begin{abstract}
This work seeks to: a) develop a concept of collaborative maturity, based on the adjusted systematization of the concept of collaborative governance elaborated by Emerson y Nabatchi (2015a), perfecting the model previously elaborated by Costa (2016), and elements and questions usually present en established models of maturity; b) operationalize the concept, through the construction of an instrument for measuring collaborative maturity; and c) to test the validity of the content of the operationalization from the semantic validity of the instrument vis-à-vis the model of Emerson. The text is divided into five parts. An introduction addresses the methodological issues that pertain to the construction of models. Part two deals with collaborative governance and presents the systematization elaborated by Emerson et al. (2012). Part three deals with maturity and presents methodological issues related to the construction of maturity models. Part four deals with the construction of the collaborative maturity model and its validation of content. The last part elaborates considerations on limitations and follow-ups.
\end{abstract}

Keywords: Collaborative maturity, governance, content validation.

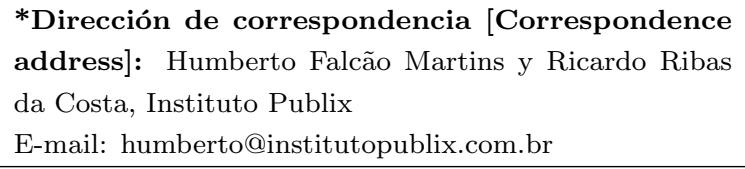




\section{Introdução ${ }^{1}$}

Os conceitos de maturidade aparecem nas ciências sociais e nas humanidades relacionados a um estado de plenitude, de preenchimento de lacunas e requisitos evolutivos. No campo da gestão, em particular gestão do desempenho, gestão de processos, gestão de tecnologia da informação, gestão de pessoas, gestão da qualidade e gestão de projetos, há múltiplos modelos de maturidade que se baseiam em escalas semânticas que definem estágios menos e mais maduros - em relação aos quais situações de fato são contrastadas e avaliadas. O grande pressuposto por detrás de escalas, mensurações e avaliações do grau de maturidade é de que há uma correlação direta positiva entre maturidade e desempenho (ou ao menos algum aspecto do desempenho ou capacidades).

A governança colaborativa, por seu turno, é uma realidade e um objeto de estudo de primeira grandeza. Sinteticamente, na definição de Emerson y Nabatchi (2015a), governança colaborativa é:

\begin{abstract}
"processes and structures of public policy decision making and management that engage people constructively across the boundaries of public agencies, levels of government, and/or the public, private and civic spheres in order to carry out a public purpose that could not otherwise be accomplished".
\end{abstract}

Há alguns elementos nesta definição que merecem ser destacados. Primeiramente, o caráter transorganizacional, o trabalho além das fronteiras, a formação de redes pluri/multi institucionais de governança (sejam entre organizações públicas, entre organizações públicas e privadas ou entre organizações privadas). Governança colaborativa é a governança em rede. Segundo, o caráter colaborativo, o engajamento construtivo inobstante as regras e estruturas de incentivos que impõem, induzem e obrigam agentes a fazer algo. Governança colaborativa é, nesse sentido, governança por propósito. Terceiro, embora menos explicito na definição apresentada, o engajamento da sociedade em rede, a sociedade conectada, no sentido de que constitui formas não institucionais de colaboração para significação, formulação, implementação e monitoramento e avaliação de políticas públicas.

\footnotetext{
${ }^{1}$ Somos gratos à Mário Woortmann, Gustavo Coelho, Renata Miranda, Jean Cançado, Carlos Barros, Marjorie Santos, Juliana Souza, Juliano Loureiro, Luciana Silva, Monique Ribeiro, Plínio Souza, Rosiane Stutz, Sérgio Deluiz, Tathiana Sampaio e Alexandre Silva pela colaboração dada na validação do questionário.
}

Como objeto de estudo, a governança colaborativa tem merecido inúmeras abordagens. Não é propósito deste artigo elaborar uma revisão de literatura detalhada, mas vale destacar algumas características da literatura a respeito do tema. Primeiramente, aspectos teóricos e estudo empíricos sobre governança colaborativa, em linha com a definição elaborada acima, aparecem já há algum tempo de forma multifacetada em múltiplas abordagens nos campos da economia (Ostrom, 1990; Fischer et al., 1981), ciência política (Olsen, 1965; Axelrod, 2006) e administração pública (Agranoff y McGuire, 2001, 2004; Pressman y Wildavsky, 1973), para citar apenas alguns. Segundo, há abordagens sistematizantes, não apenas no sentido de encetarem uma revisão sistemática de literatura, mas de proporem sistematizações conceituais a partir de abordagens muito diversificadas, embora em alguma extensão sobrepostas. Dentre estas, duas destacam-se: Ansell y Gash (2008) e Emerson et al. (2009).

A proposta de Emerson et al. (2012), além de mais recente, avança sobre o de Ansell y Gash (2008) em termos de abrangência da sistematização, incorporando novas abordagens ${ }^{2}$, e proposta metodológica mais avançada, na medida em que propõe um modelo (a partir de dimensões e variáveis e indicação de "mecanismos causais) e elabora hipóteses a respeito. Por estas razões, a abordagem de Emerson e outros torna-se mais operacionalizável, permitindo, assim, submeter seu modelo à testes de validade.

Do ponto de vista metodológico, a grande questão dos modelos é testar sua validade e esta só pode ser amplamente verificada quando o construto/modelo é mensurado/operacionalizado. Segundo Adcock y D., 2001, a conceptualização e mensuração se coloca em quatro níveis: a) o conceito antecedente, com uma constelação de significados potencialmente diversos; b) o conceito sistematizado, com uma formulação explícita específica do conceito; c) operacionalização propriamente dita, com métricas a partir de definições operacionais e indicadores, com pontuações sistemáticas que podem variar de medidas simples a índices agregados complexos; e d) aplicação da operacionalização em casos específicos, gerando-se os indicadores ou classificações qualitativas.

\footnotetext{
${ }^{2}$ Relativamente à: "colaboração intersetorial (Bryson et al., 2006), planejamento colaborativo (Bentrup, 2001; Innes y Booher, 1999; Selin y Chevez, 1995), processos de colaboração (Daniels y Walker, 2001; Ring y Van de Ven, 1994; Thomson y Perry, 2006; Wood y Gray, 1991), gestão de redes (Koppenjan y Klijn, 2004; Milward y Provan, 2000), gestão pública colaborativa (Agranoff y McGuire, 2001; Cooper et al., 2006; Leach, 2006), governança ambiental e resolução de conflitos (Agrawal y Lemos, 2007; Emerson et al., 2009), e governança colaborativa (Ansell y Gash, 2008)" (Emerson et al., 2012).
} 
Os autores propõem três tipos de validação: a) de conteúdo, onde é avaliada a extensão na qual os indicadores captam as dimensões e atributos do conceito sistematizado, sem omissões ou inclusões inadequadas, assumindo-se que o conceito sistematizado guarda coerência com o conceito antecedente; b) convergente/discriminante, onde, a partir da aplicação das métricas, é avaliada a extensão na qual os indicadores utilizados estão de fato mensurando o conceito sistematizado (convergência) ou outro conceito (discriminante); e c) nomológica, onde avalia-se a extensão na qual as hipóteses do modelo/construto/conceito, as relações causais entre variáveis, são corroboradas. Adcock y D., 2001 Validações de conteúdo e convergente/discriminante apoiam inferências descritivas; validação nomológica apoia inferência causal.

Os objetivos deste trabalho são: a) desenvolver um conceito de maturidade colaborativa, a partir da sistematização ajustada do conceito de governança colaborativa elaborada por Emerson e outros, aperfeiçoando o modelo anteriormente elaborado por Costa (2016), e de elementos e questões usualmente presentes em modelos consagrados de maturidade (em particular de gestão de tecnologia, processos, projetos, qualidade e desempenho); e b) operacionalizar o conceito, por meio da construção de um instrumento de mensuração da maturidade colaborativa; e c) testar a validade de conteúdo da operacionalização a partir da validade semântica do instrumento vis-à-vis o modelo de Emerson.

Este artigo se encarta em um projeto de pesquisa com objetivos mais amplos e ousados que buscarão, em etapas ulteriores: a) testar a validade convergente/discriminante, a partir da aplicação do instrumento e de análise de componentes principais e teste de Cronbach; b) testar a validade nomológica do modelo, a partir de análise de correlações destinadas a testar hipóteses formuladas por Emerson e outros; e c) possibilitar a aplicação do modelo de maturidade colaborativa para a formulação de hipóteses sobre determinantes do desempenho de regimes de colaboração. Ademais, acredita-se que a aplicação do modelo, por si só, poderá proporcionar inputs para tomada de decisão e ativação de atitudes de melhoria de desempenho em regimes colaborativos - como creem Bititci et al. (2015).

O texto está divido em outras quatro partes, além desta introdução. A parte dois trata de governança colaborativa e apresenta a sistematização elaborada por Emerson e outros. A parte três trata de maturidade e apresenta questões metodológicas relacionadas à construção de modelos de maturidade. A parte quatro trata da construção do modelo de maturidade colaborativa e sua validação de
Figura 1: Modelo de Governança colaborativa de Emerson e outros

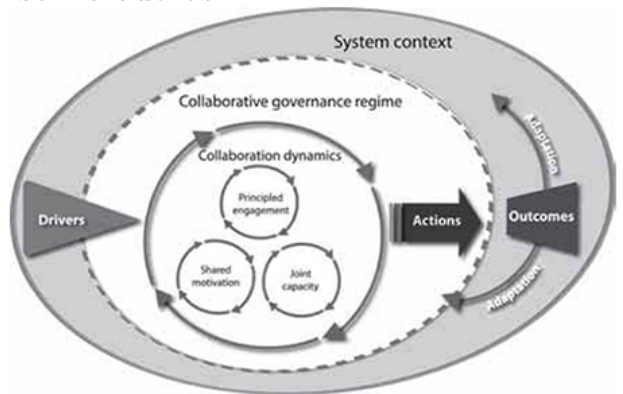

Fonte: Emerson y Nabatchi (2015a)

conteúdo. A última parte elabora considerações sobre limitações e prosseguimentos.

\section{Governança colaborativa: o modelo de Emerson e outros}

No artigo "An Integrative Framework for Collaborative Governance" (Emerson et al., 2012) e no livro "Collaborative Governance Regimes" (Emerson y Nabatchi, 2015a), os autores desenvolvem uma sistematização de "governança colaborativa", a partir de um amplo conjunto de abordagens teóricas, pesquisas de campo e conhecimentos provenientes da prática. A definição já mencionada engloba

\begin{abstract}
"'multipartner governance', which can include partnerships among the state, the private sector, civil society, and the community, as well as joinedup government and hybrid arrangements such as public-private and private-social partnerships and co-management regimes (Agrawal and Lemos 2007)".
\end{abstract}

(Emerson et al., 2012).

A sistematização foi elaborada sob a forma de um modelo de governança colaborativa, cujos elementos estão ilustrados na Figura 1 e serão apresentados a seguir.

A arquitetura geral do modelo assemelha-se muito a um modelo sistêmico linear genérico de entradas (inputs, no caso específico, os iniciadores/direcionadores colocados pelo contexto, tais como liderança, incentivos consequenciais, interdependência e incerteza), processamento (denominado dinâmica da colaboração, que por sua vez se baseia na atuação conjunta autoretroalimentada de três componentes: engajamento com princípios, motivação compartilhada e capacidade 
para atuação conjunta), saídas (outputs, no caso as ações desenvolvidas de forma colaborativa), impactos (outcomes, tanto no contexto quanto na dinâmica colaborativa) e feedbacks (no caso das ações para a dinâmica colaborativa e para a adaptação ao contexto), formando um regime de governança colaborativa que operam num determinado contexto (a partir de fatores políticos, legais, socioeconômicos, ambientais e outros que afetam e são afetados pelo regime de governança colaborativa).

O conceito de regime de governança colaborativa é central no modelo de Emerson e outros porque denota um "modo de decisão pública no qual a colaboração transorganizacional representa o padrão predominante de comportamento e atuação".

Também é central o conceito de dinâmica colaborativa, com seus componentes interativos: engajamento com princípios, motivação compartilhada e capacidade para atuação conjunta. Estes três componentes, que podem ser vistos como processos, operam de forma interativa e contínua para gerar atuação colaborativa (como outputs) - inclusive a forma como estão representados, por engrenagens, dá a ideia de sincronismo e concomitância, não de sequenciamento linear.

A Tabela 1 apresenta um detalhamento das variáveis que compõem o modelo de governança colaborativa de Emerson e outros. O textos do artigo (Emerson et al., 2012) e do livro (Emerson y Nabatchi, 2015b) apresentam uma descrição detalhada de cada uma das variáveis apresentadas a partir de indicações de conceitos e estudos a elas relacionadas no campo da governança colaborativa. De forma a se evitar a transcrição de longos trechos do texto original de Emerson e outros, optamos por apresentar a especificação destas variáveis na Tabela 2 mais adiante no quarto segmento deste artigo.

Nenhuma sistematização será exaustiva e as mais abrangentes poderão ser problemáticas por incluir muitas dimensões enquanto que as mais focadas ou setorizadas imporão risco de reducionismo. O modelo de Emerson e outros é uma excelente sistematização do conceito de governança colaborativa. Primeiro porque é abrangente (relativamente à diversidade do conceito antecedente, no sentido de que "synthesizes and extends a suite of conceptual frameworks, research findings, and practice-based knowledge into an integrative framework for collaborative governance... [and] synthesizes and extends a suite of conceptual frameworks, research findings, and practice-based knowledge into an integrative framework for collaborative governance [...] The framework provides a broad conceptual map for situating and exploring components of cross-boundary governance systems that range from policy or program based intergovernmental cooperation to place-based regional collaboration with nongovernmental stakeholders to public-private partnerships".

Segundo, porque é genérico, no sentido de não ser setorizado,

\begin{abstract}
"integrates knowledge about individual incentives and barriers to collection action, collaborative social learning and conflict resolution processes, and institutional arrangements for cross-boundary collaboration. It is presented as a general framework that might be applied to analyses at different scales, in different policy arenas, and varying levels of complexity"
\end{abstract}

E terceiro porque apresenta um mecanismo causal que embasa hipóteses, possibilitando que seja operacionalizado e testado.

\section{Maturidade}

Maturidade é um termo muito utilizado no campo profissional e acadêmico da gestão, particularmente nas áreas de gestão de processos (Tarhan et al., 2016), gestão da qualidade (Boughzala y De Vreede, 2015), gestão de projetos (Costa y Ramos, 2013), gestão do desempenho e também no campo da TI (Bititci et al., 2015). Estima-se que a origem de tais modelos tenha sido o Quality Management Maturity Grid (QMMG) de Crosby, 1979, criado para avaliar a adesão de empresas aos princípios da gestão da qualidade; assim como, posteriormente, modelos de maturidade na área de tecnologia da informação, destacadamente o Capability Maturity Model (CMM) ara engenharia de software na década de 60 (Boughzala y De Vreede, 2015). 


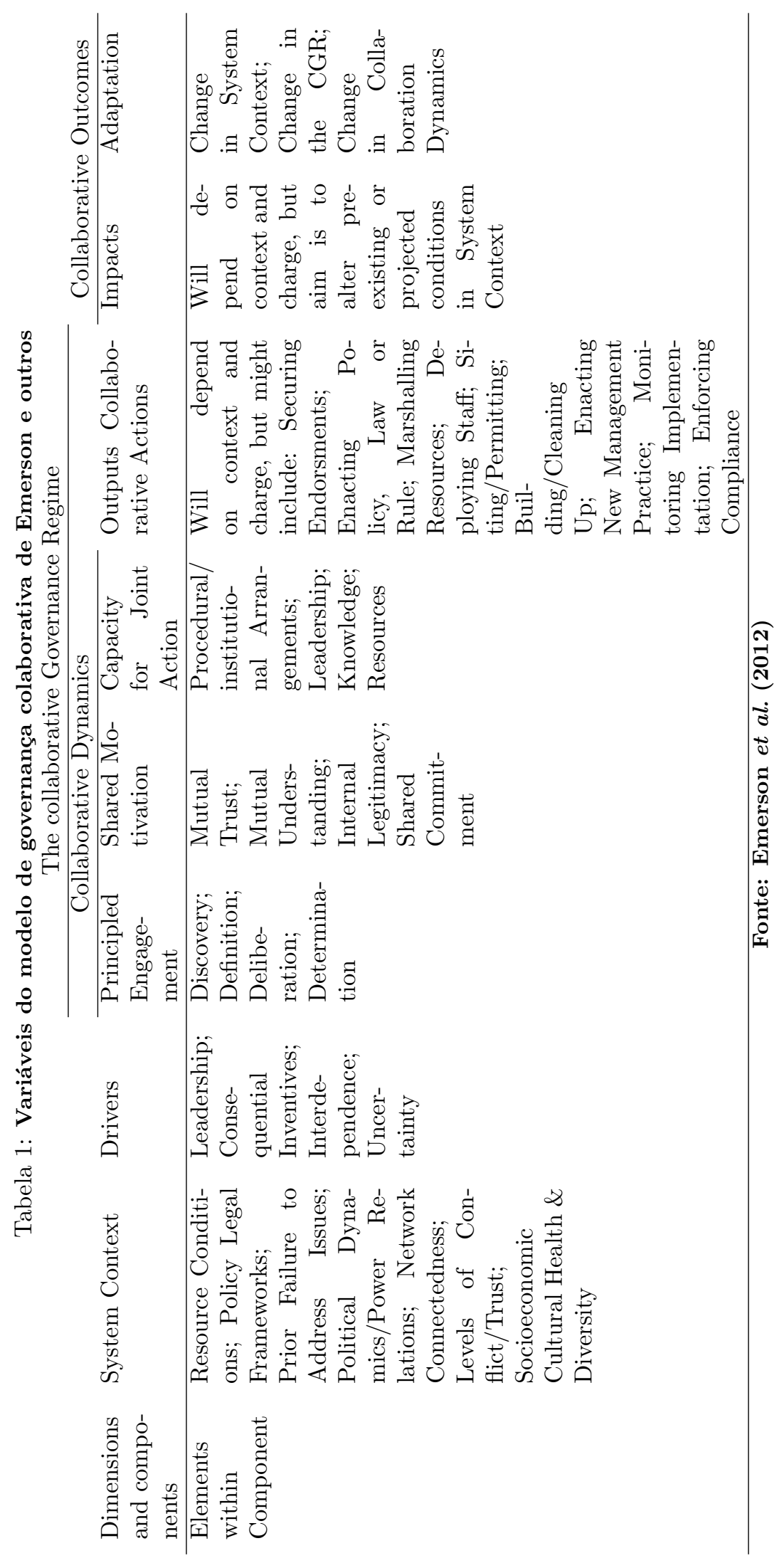


Muitos modelos de maturidade citam como referência a Teoria da Maturidade-Imaturidade, de Chris Argyris (1969), que se refere a indivíduos e que muitos transpõem para organizações:

\footnotetext{
"a mature person is characterized for being active, independent, self-confident and self-controlled; on the contrary, an immature person is passive, dependant, has lack of confidence and feels need of control by others".
}

Atribui-se à origem em Argyris de muitas derivações do conceito de maturidade, seja a ideia de plenitude, de preenchimento de lacunas e requisitos evolutivos que contribua para um estado melhor. Entretanto, não há um acordo a respeito de plenitude de que e para que finalidade, noções estas definidas de forma específica no âmbito de cada modelo de maturidade.

Fraser et al. (2002) afirmam que maturidade carrega um significado intrínseco de "perfeição",

\begin{abstract}
"conveying the notion of development from some initial state to some more advanced state. Implicit in this is the notion of evolution or ageing, suggesting that the subject may pass through a number of intermediate states on the way to maturity of maturity, with a description of characteristic performance at various levels of granularity".
\end{abstract}

A qualidade de maduro varia de acordo com múltiplos objetos e casos. No que se refere aos muitos modelos de maturidade nas áreas descritas acima, maturidade está associada à conformidade a um modelo de maturidade que define práticas e atributos em diferentes níveis do que considera menos e mais maduro.

Para Dooley et al., 2001 e o CMMI Product Team (2002), a maturidade é o grau com que um processo ou atividade é institucionalizado e efetivado por toda a organização. Desta forma, o nível de maturidade é um indicativo da sofisticação, da estabilidade e frequencia com que práticas, técnicas e procedimentos padrão relacionados a uma área especifica são adotados (Jucá Jr y Amaral, 2005).

Para uma série de modelos de maturidade em gestão de processos (De Bruin y Rosemann, 2005; Rosa dos Santos, 2003; Siqueira, 2017), tecnologia (Dourado, 2017), projetos (Brookes y Clark, 2009; PM Solutions, 2017), desempenho (Spitzer, 2007) e pessoas (Curtis et al., 2009) é muito comum a definição de estágios baseados em noções de níveis de completude e frequência, em ordem crescente de maturidade, relacionados à:
1. Métodos, processos, diretrizes não definidos, repetitivas, incipientes ou eventuais;

2. Métodos, processos, práticas, diretrizes definidos;

3. Métodos, processos, práticas, diretrizes definidas, controladas, previsíveis, geridas;

4. Métodos, processos, práticas, diretrizes em constante melhoria gerando transformações visíveis.

Fraser et al. (2002), tomando processos de trabalho como objeto, desenvolveram uma tipologia de modelos de maturidade que inclui:

1. grades maturidade, onde as linhas contem um conjunto de práticas/atividades e as colunas contem uma descrição dos níveis de maturidade. "The principal idea of the maturity grid is that it describes in a few phrases, the typical behaviour exhibited by a firm at a number of levels of 'maturity', for each of several aspects of the area under study. This provides the opportunity to codify what might be regarded as good practice (and bad practice), along with some intermediate or transitional stages... Maturity grids tend to trade resolution (in terms of number of levels) against richness of description.." (Fraser et al., 2002)

2. modelos de maturidade de capacidade, que especificam um conjunto de práticas chave para o alcance de um objetivo e as colunas contem uma descrição geral de maturidade para o conjunto, não por prática;

3. questionário com escala likert, onde a questão é o enunciado de uma boa prática e o respondente pontua a maturidade conforme a aderência da questão. "Multi-point likert scale could be regarded as a maturity grid but with only one extreme level explicitly described (usually the top level), and other simplied by anchors such as 'strongly disagree', 'disagree' and so on. It is left to the respondent to interpolate without further guidance." (Fraser et al., 2002)

A maioria dos modelos de maturidade define estágios idealizados e os caracteriza por meio de escalas semânticas; muito mais que o grupamento de categorias encontradas por meio de observação (Lahrmann et al., 2011). Aparentemente, o desenvolvimento de modelos e instrumentos de mensuração de níveis de maturidade possui mais apelo que o teste de qualidade dos modelos subjacentes, 
no sentido de se verificar se de fato ensejam operacionalizações válidas de conceitos bem sistematizados.

Há muitas formas e propósitos de aplicação de modelos de maturidade: autoaplicação, individual ou em grupo; ou assistida por especialista. A grande questão da aplicação de modelos de maturidade é a necessidade de redução de viés e de objetivação, posto que as avaliações são na maioria das vezes qualitativas e baseadas em percepções - por mais fundamentadas que sejam em evidências.

Há também propósitos de auditoria e certificação, muitas vezes vinculados a incentivos meritórios ou pecuniários, e outros de aprendizado e melhoria. Independentemente do propósito ou do método de aplicação, há evidencias de que o efeito de discussão em grupo já gera benefícios (Chiesa et al., 1996).

Há muito pouco sobre metodologias de construção de modelos de maturidade.

\footnotetext{
"How are these grids constructed? The number of levels is to some extent arbitrary, and depends on the ability to identify suitable labels or descriptive text which clearly differentiate one level or stage from the next.[...] grid-based tools tend to contain 4 levels, and some even contain 3".
}

(e.g. Macbeth y Ferguson, 1994).

Becker et al., 2009 partem de princípios da ciência do design (Hevner et al., 2004), voltada à melhoria de capacidades de solução de problemas por meio da criação de artefatos inovadores, tais como construtos, modelos, métodos e experimentação/prototipação (March y Smith, 1995), para estabelecer 8 requisitos para a construção de modelos de maturidade:

1. Comparação com outros modelos existentes. "The new model may also just be an improvement of an already existing one" (Zelewski, 2007: 93-98);

2. Procedimento iterativo. O desenvolvimento do modelo deve seguir um passo-a-passo, podendo ocorrer a repetição de passos;

3. Avaliação. Inclui a avaliação de princípios, premissas, utilidade, qualidade e efetividade de forma iterativa e com rigor científicometodológico. "Evaluation of all (intermediary) results must be carried out with appropriate scientific methodology. Since different methods may be used in the development of the artifact and in the evaluation of the particular results, design science characteristically adopts a multi-methodological procedure." (Hevner et al., 2004);

4. Procedimento multi-metodológico. O emprego de uma variedade de métodos fundamentados e alinhados (March y Smith, 1995) traz dificuldades e sugere um enfoque ontológico (Frank, 2004);

5. Identificação da relevância do problema. É preciso demonstrar a relevância da solução ao problema que o modelo de maturidade trará por intermédio de pesquisadores ou praticantes;

6. Definição do problema. O domínio de aplicação do modelo de maturidade, as condições de aplicação e os benefícios almejados devem estar claros antes da sua concepção;

7. Apresentação de resultados direcionada aos usuários. A apresentação do modelo de maturidade deve ser direcionada às necessidades dos seus usuários;

8. Documentação científica. Necessidade de documentação integral do processo de concepção, considerando cada etapa, partes envolvidas, métodos e resultados.

Estes requisitos foram tomados como recomendações: houve identificação de modelos congêneres e comparação entre eles; as etapas de operacionalização foram iterativas, no sentido de que geraram e incorporaram ajustes; com método e rigor científico em todas as suas etapas; com resultados potencialmente relevantes para praticantes e acadêmicos; e de forma documentada.

No caso da maturidade colaborativa, optou-se pela construção de uma grade de maturidade baseada na frequência e intensidade/plenitude (grau de implementação) com que os elementos do regime de colaboração, conforme propostos por Emerson se apresentam.

\section{Construção do modelo e va- lidação de conteúdo}

Um modelo de maturidade colaborativa busca mensurar o grau de maturidade (frequência e intensidade) dos elementos de um regime de governança colaborativa (conforme definido por Emerson). A construção do modelo, operacionalizando o conceito de regime de governança colaborativa, seguiu os seguintes passos: 
1. Especificação das variáveis do modelo de Emerson e seus atributos (Tabela 3);

2. Especificação dos atributos de maturidade;

3. Definição da forma de operacionalização dos atributos de maturidade relativamente aos atributos do regime de governança colaborativa.

Os atributos obtidos a partir de Emerson e outros passaram por uma tradução rigorosa para o português (na qual se buscou preservar o sentido no texto original e sua compreensão no idioma português do Brasil) e os enunciados representam ajustes semânticos com o intuito de recuperar/aclarar o sentido de modo a serem convertidos em itens de questões.

Cabem três observações em relação aos ajustes. Primeiramente, uma questão de delimitação: optou-se por suprimir as variáveis de contexto. Originalmente, Emerson definiu o regime de governança colaborativa como parte do contexto e não o contexto como parte do regime. Embora variáveis contextuais possam, numa perspectiva sistêmica, ter um caráter determinístico, incluí-lo implicaria em avaliar a maturidade do contexto e hipotetizar sobre relação maturidade contexto e do regime de governança colaborativa. Emerson aborda questões contextuais mas não trata o contexto com maior abrangência e profundidade. Apenas lembra que há um contexto. Tratar o contexto como sugerido aumenta muito a complexidade do modelo, podendo ser objeto de uma outra empreitada.

Segundo, buscou-se dar um tratamento mais explícito à dimensão cultura organizacional, que embora esteja claramente subjacente às demais, pode receber um tratamento mais sistemático como componente da capacidade de atuação conjunta muito inspirado no conceito de colaborarquias autogeridas de Agranoff (2007).

Terceiro, a vinculação da categoria impactos/adaptação ao conceito de valor público a partir de Moore $(1995,2011)$, não apenas abordando impactos, mas também a extensão na qual os impactos foram valorados como satisfatórios pelos beneficiários em questão - esta questão aparecerá na versão final do questionário.

Os atributos de maturidade são intensidade e frequência. Logo, considera-se mais madura a experiência colaborativa que apresentar os componentes do modelo de governança colaborativa de forma mais frequente e intensa. A frequência é uma proxy de habitualização, sugerindo um processo de institucionalização baseado em objetivação, habitualização e sedimentação (Tolbert y Zucker, 1999).
A intensidade é uma forma de se abordar o atributo seminal da plenitude, em linha com a maioria dos modelos de maturidade no campo da administração.

A principal questão aqui é qual é melhor forma de mensurar cada atributo, questão esta que, neste caso, se reduz a duas principais alternativas: escala likert ou escala semântica (grade) - assumindo-se que estas opções são superiores a outras formas tais como notas atribuídas pelos respondentes.

As escalas são formas mais precisas de operacionalização, no caso, em se tratando de variáveis qualitativas, escalas semânticas.

\begin{abstract}
"Las escalas garantizan mejor la ordinalidad al considerar las estructuras entre los Indicadores. Las reactivos de una medición combinada pueden tener diferentes intensidades en términos de la variable. (...). En general, las escalas son superiores a los índices porque toman en cuenta la intensidad con que los reactivos reflejan la variable que se mide. (...) Tanto el formato de Likert como el diferencial semántico tienen más rigor y estructura que otros formatos de preguntas. Como dijimos. estos formatos arrojan datos adecuados lo mismo para índices que para escalas. (...) En este punto debemos recordar que una de las principales funciones de la construcción de una escala es la reducción eficaz de los datos. Las escalas ofrecen una técnica para presentar los datos en forma resumida al tiempo que conservan tanta información original como sea posible".
\end{abstract}

(Babbie, 2000: 148-164).

A questão é que a adoção da escala semântica como alternativa de operacionalização implicaria, em princípio, na construção de uma escala semântica para cada um dos atributos da governança colaborativa, vezes dois atributos da maturidade, resultando num instrumento com cerca de uma centena itens de resposta, excessivamente complexo. A solução encontrada foi a construção de uma matriz semântica simplificada, com enunciados sintéticos, em palavra única, dispostos em sequência de valoração crescente (como que representando um contínuo ordinal de 0 a 3 , deliberadamente sem opção central, de modo a evitar a tendência de centro) e em blocos paralelos de atributos de maturidade que possam ser vistos lado a lado na sequencia dos atributos de governança colaborativa.

Dessa forma, para cada atributo do modelo corresponde uma questão (item de resposta), com cinco opções para cada atributo de maturidade: 
Tabela 2: Especificação detalhada das dimensões, variáveis e atributos do modelo de Emerson, com ajustes

\begin{tabular}{|c|c|c|}
\hline Dimensōes & Variáveis & Atributos \\
\hline \multirow{7}{*}{ 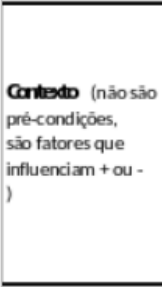 } & Recursos & A disponibilidade / facilidade de acesso à recursos (financeiros, humanos, tecnológicos etc.) áteta a colaboraçāo \\
\hline & \begin{tabular}{|l|l|l|l} 
Marcolegal \\
Legado de falhas
\end{tabular} & 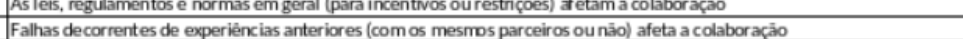 \\
\hline & Dinâmica politica / relaçōes de poder & $\begin{array}{l}\text { A dinâmica pol itica (no sentido do sistema politico e partidário) e relaçöes de poder (incluindo tanto as diversas esferas de } \\
\text { poder: municipal, estadual e federal, quanto os diversos sctores: privado, público e terceiro setor) afetam a colaboraçáo }\end{array}$ \\
\hline & \begin{tabular}{|l} 
Conectividade da rede \\
\end{tabular} & O grau préexistente de conectividade dentro da rede (com parceiros jả estabelecidos) e com outras redes afeta a \\
\hline & Niveis de conflito/confiança & Os niveis históricos de conflito em torno de interesses bem identificados afeta a col abor açäo \\
\hline & Diversidade sócio-econômica & $\begin{array}{l}\text { Os niveis históricos de confiança nos relacionamento afetam a coläboraçáo } \\
\text { A diver sidade económica (renda, rigueza) e social (etrica cultural, educacional, condicōes de bemestar etc.) pode đátar a }\end{array}$ \\
\hline & Retroalimentaçäo do contexto & $\begin{array}{l}\text { Os impactos produzidos pelo regime de colaboraçào sầ capazes de afetar/modificar (positva ou negativamente) o próprio } \\
\text { contexoem que se estabelecem }\end{array}$ \\
\hline \multirow{7}{*}{ Dredanadares } & \multirow{3}{*}{$\begin{array}{l}\text { Udearanga (presença de um lider em } \\
\text { condiçoes de iniciar a iniciativa e buscar } \\
\text { recursos) }\end{array}$} & Há boa vontade/disposiçāo do lider para abr açå uma causa coletiva (e nāo particular) \\
\hline & & Líder è Imparcial em relaçảo às preferências dos envolvidos \\
\hline & & Hả boa vontade/disposiçāo do lider para atuar na provisāo de equipes, tecnologia e outros recursos necessários \\
\hline & \multirow{2}{*}{ Incertivos para colaborar } & $\begin{array}{l}\text { Forças e fraquezas comuns (passiveis de controle): Fatores internos, negativos ou não, tais como necessidade de recursos } \\
\text { interesses ou oportunid ades, percebidos pelas partes mutuamente interessad as na soluçăo de um determinado problema, } \\
\text { serviram de motivo para o inicio do regime de colaboraçäo }\end{array}$ \\
\hline & & 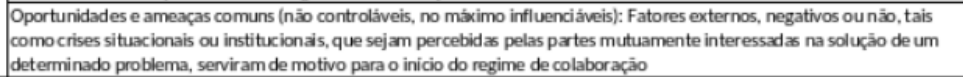 \\
\hline & hterctpentênda & $\begin{array}{l}\text { As partes envolvidas no regime de colaboraçäo entenderam que nảo seriam capazes de de solucionar o problema por ś sós, } \\
\text { ou seja, individualmente }\end{array}$ \\
\hline & hartez & Partes interessadas sentem-se incapzes de lidar por si só com as incertezas emvolvendo a solucảo do problema comum \\
\hline
\end{tabular}

Fonte: Elaboraçao própria com base em Emerson et al. (2012)

Tabela 3: Especificação detalhada das dimensões, variáveis e atributos do modelo de Emerson, com ajustes (continuaçao)

\begin{tabular}{|c|c|c|c|}
\hline & & Descoberta & Houve identificaçăa/revelasço de interesses, preocupaçōes, valores individuais ou compartihudos que ensejasse o inicio do \\
\hline & & Descoberta & 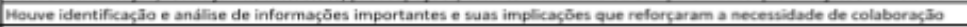 \\
\hline & & & $\begin{array}{l}\text { Hé esforço continuado de articulaçăo de propóstos e objetivos comuns para construça de sienificados compartihados entre } \\
\text { os interessados no regime de colaboraço }\end{array}$ \\
\hline & & Definisabo & 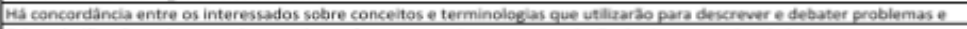 \\
\hline & Engajamento com & & 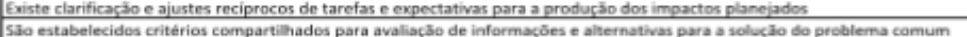 \\
\hline & principios & & $\begin{array}{l}\text { O processo deliberativo permite defesa aberta (direito à voz) e qualficada de interesses individuais (incluindo exame } \\
\text { ponderado de questoes) e de terceiros (incluindo escutas de outras perspectivas) }\end{array}$ \\
\hline & & Deliberasaso & 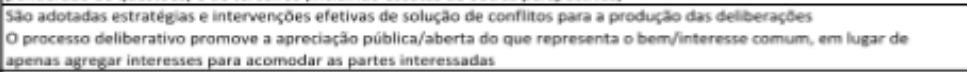 \\
\hline & & & O processo decisório é estruturado e inclui a formulaçao de apendas, discussibes, erupos de trabathe etc. \\
\hline & & Determinasăo & O processo decisório em vigor conduz à determinaçbes substantivas tais como acordos, asbes, recomendasbes etc. \\
\hline & & Conflanga mütua & 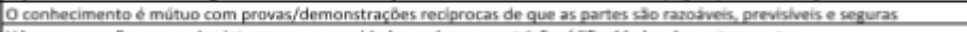 \\
\hline & & Entendimento mùtuo & 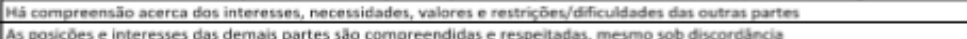 \\
\hline & $\begin{array}{l}\text { Motivaçă } \\
\text { compartilhada }\end{array}$ & Legitimidade interna & 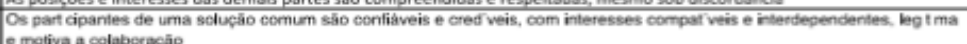 \\
\hline Dinàmica & & \begin{tabular}{|l|} 
Comprometimento \\
cemeantilhade
\end{tabular} & $\begin{array}{l}\text { As partes salo capazes de cruzar as ironieiras organizadionais, seloriais ou jur solic onais que os separavam e de } \\
\text { comprometerem se com um cam nho compartilhado }\end{array}$ \\
\hline Colaborativa & & \begin{tabular}{|l|} 
Arranjios \\
institucionais/ \\
procedimentais fintra
\end{tabular} & 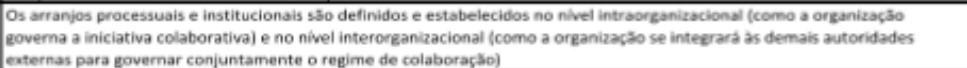 \\
\hline & & interorzaniracionais) & $\begin{array}{l}\text { A estrutura de autoridade interna das instituicōes colaborativas tende a ser menos hierárquica e estivel, e mais complexa e } \\
\text { flülda que as estruturas das burocracias tradicionais }\end{array}$ \\
\hline & & Lideransa & 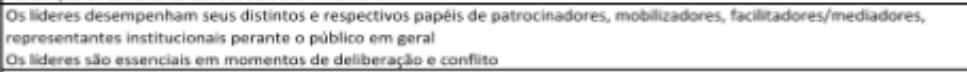 \\
\hline & & & Os lideres asseguram a implementaçāo das determinaçōes colaborativas \\
\hline & Capacidade de & Conhecimento & $\begin{array}{l}\text { O capital social de conhecimento compartilihado é resultante da pesagem, processamento e integrasio com os valores e } \\
\text { julpamentos de todos os participantes do repime de colaboraço }\end{array}$ \\
\hline & & Cultura organitracional & 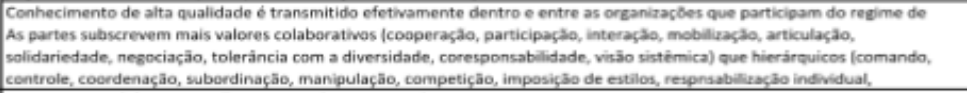 \\
\hline & & & Eviste potencial de compartihamento e alavancagem de recursos escassos \\
\hline & & & Ha disponiballdade de recursos financeiros \\
\hline & & Recurnos & 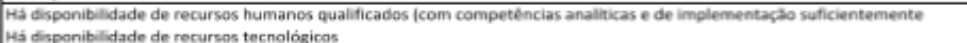 \\
\hline & & & His disponiblildade de recursos logisticos (materiais, equipamentos, instalaçles) \\
\hline & & & Há disponibilidade de recursos de poder (meios coefcitivos, normativas e regulatórios) \\
\hline Açles colaborativas & & & 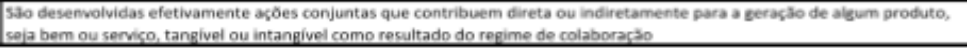 \\
\hline Impactos/Adaptas & Criaçăo de valor pü & & $\begin{array}{l}\text { Constata-se efetivamente uma mudança de estado naquilo que tinha que tinha que ser feito e/ou se planieva fazer, como } \\
\text { resultado das açes conjuntas }\end{array}$ \\
\hline & Aprimoramento da & imanca colaborativa & A atuaçào colaborativa tem gerado mudanças positivas no próprio regime de colaboraçao \\
\hline
\end{tabular}

Fonte: Elaboraçao própria com base em Emerson et al. (2012) 
1. relativamente à intensidade, quatro opções relativas à escala de significância (insignificante, mal se nota, perceptível e contundente) e uma opção "não se aplica"; e

2. relativamente à frequência, quatro opções relativas à escala de frequência (nunca, algumas vezes, frequentemente e sempre) e uma opção "não se aplica".

A definição da forma de operacionalização dos atributos de maturidade relativamente aos atributos do regime de governança colaborativa estão na Tabela 3, formato este que corresponde, praticamente, à forma de apresentação do instrumento de mensuração a ser aplicado - que, em formato eletrônico, deverá disponibilizar, ao se clicar as células de respostas, as opções aludidas para que o respondente possa marcá-las. Observa-se ainda que aos atributos de cinco variáveis (incentivos para colaborar, interdependência e descoberta, criação de valor público e aprimoramento da colaboração) não se aplica o atributo de maturidade "frequência", uma vez que as variáveis referem-se à eventos circunscritos temporalmente no passado.

O propósito da validação é fazer com que a mensuração reflita o significado real do conceito em questão. A validação de conteúdo é uma validação semântica ou de face para assegurar a qualidade:

1. dos termos, se são fiéis às variáveis conforme especificadas e representam uma linguagem adequada;

2. da sintaxe, ou da disposição dos termos (verbos e seus tempos, objetos) nas sentenças para expressar os significados; e

3. da semântica, em última análise o significado, a extensão na qual representam ou denotam o modelo sob operacionalização ou o alinhamento entre a conceituação, a definição nominal e a definição operacional (Babbie, 2000).

A validação de conteúdo se deu de duas formas: painel de especialistas e aplicação piloto em 12 casos. A primeira consistiu na formação de um grupo focal com especialistas em gestão do desempenho e em gestão colaborativa, grupo este que após a leitura do texto de Emerson e outros (2011), participou de uma discussão item a item, buscando aferir a aderência das sentenças ao modelo em termos de adequação de termos e sintaxe, análise psicométrica para identificação de questões com duplo sentido (double-barreled), sentido dúbio (confusing) e viés de resposta (leading questions) e análise de diferencial semântico das opções (a extensão na qual são bem entendidas e efetivamente diferenciam). As sugestões do grupo focal implicaram na revisão de $90 \%$ das questões e na criação de duas novas questões, desdobradas de questões consideradas de duplo sentido. Não houve alterações nas opções de resposta.

A segunda validação foi baseada na aplicação piloto de um instrumento derivado da primeira especificação dos atributos das variáveis em 12 casos seletos para teste de fadiga e registro de dúvidas e sugestões dos aplicadores e participantes. Inicialmente, o instrumento foi concebido para se aplicado de forma individual ou grupal, embora referenciada a um mesmo caso - a experiência colaborativa ou o regime de governança colaborativa que é a unidade observacional; portanto, é preferível que o instrumento seja aplicado para distintos participantes que representem a pluralidade de partes envolvidas em um regime de governança colaborativa. No que concerne à aplicação, as questões fundamentais são proporcionar uma compreensão homogênea entre os aplicantes e lastrear as respostas em evidências de modo a se promover objetivação em detrimento de subjetividade. Assim, aplicações orientadas em grupos representativos (distintos participantes que representem a pluralidade de partes envolvidas), precedidas de explicações, tendem a ser superiores a auto-aplicações individuais, embora estas últimas também sejam válidas em termos do potencial de geração de reflexões sobre melhorias necessárias. Em todo caso, a validação convergente/discriminante poderá testar a qualidade de distintas formas de aplicação.

Também é possível que o questionário seja derivado em outros instrumentos mais simples, a partir da agregação de questões em torno das variáveis do modelo de Emerson e outros; ou mesmo modelos mais detalhados, com uma escala semântica mais especificada e exigência de registro de evidências. O uso poderá determinar distintas derivações desta forma proposta de operacionalização do conceito de governança colaborativa, se com finalidade de pesquisa, para efeito de capacitação ou consultoria.

Uma ultima questão em relação à operacionalização é a mensuração, propriamente dita, no sentido de que a aplicação do instrumento permitirá a conversão de variáveis nominais em medidas ordinais intervalares (na medida em que à cada escolha nas escalas de frequência e intensidade de cada item se associem valores), no sentido de se gerar um índice de maturidade colaborativa. Há, nesse sentido, questões metodológicas que devem ser tratadas de antemão, previamente à aplicação, e outras que requererão aplicação em maior escala - embora o instrumento permita uma aplicação totalmente qualitativa. 


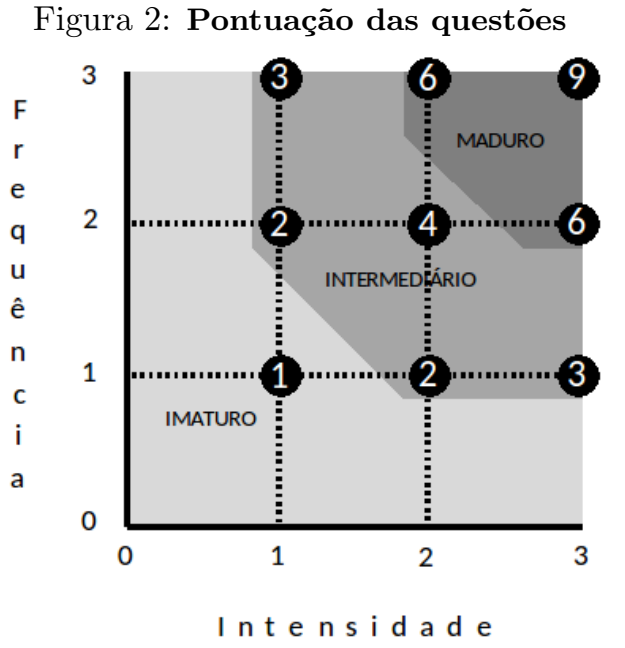

No que se refere à pontuação de cada questão propõe-se uma escala quali-quant bidimensional (a partir dos dois atributos de maturidade: frequência e intensidade) na qual cada dimensão varia de 0 (zero) a (três) e a pontuação da questão corresponde à multiplicação das notas das duas dimensões, conforme ilustrado na Figura 3, indicando as faixas de maturidade "maduro", "intermediário" e "imaturo". As questões cuja dimensão frequência não é contada pontuam-se pela nota da dimensão intensidade multiplicada por três; e aquelas que possuem opções "não se aplica" marcadas deverão ser expurgadas no computo final.

Dessa forma, o índice de maturidade colaborativa seria a média aritmética simples das pontuações dos seis grandes blocos do modelo de Emerson e outros (2011; 2015): direcionadores, engajamento com princípios, motivação compartilhada, capacidade para atuar de forma colaborativa, ações colaborativas e impactos/adaptação. As pontuações destes blocos corresponderiam, por seu turno, à média aritmética simples das pontuações das respectivas questões válidas (excluídas as opções "não se aplica"). Ao final, tem-se um índice que varia de 0 a 9 (podendo ser convertido em escalas de 0 10 ou $0-3)$ e permite o enquadramento de um regime de governança colaborativa em três faixas de maturidade interpoladas "maduro" (6 a 9 pontos), "intermediário" (2 a 4 pontos) e "imaturo" (0 a 1 ponto), também ilustrados na Figura 3. O caráter intervalar das faixas é muito mais útil no sentido de proporcionar discussões em torno de ações de melhoria para maturação do que um número absoluto para efeito de ranqueamento.

Outras questões metodológicas relacionadas à mensuração só poderão ser ajustadas após a aplicação em maior escala, no sentido de que técnicas estatísticas tais como análises de com- ponentes principais e teste de Cronbach poderão, em alguma extensão, proporcionar a realização de testes de validade lógica (se os atributos certos estão incluídos), de unidimensionalidade (se não se medem outros conceitos), de generalidade/especificidade (se não se identificam subtipos), de variabilidade (que valores indicam a existência do fenômeno) e bivariabilidade (se há relações bivariadas entre os itens). Tais verificações poderão também ser sugestivas de ponderação entre os blocos ou questões.

\section{Considerações finais}

Esta proposta é parte de um projeto de pesquisa mais amplo que busca investigar a relação entre maturidade colaborativa e desempenho de regimes colaborativos. Nesse sentido, representa um pequeno, porém primeiro e significativo passo nesta direção. Além de possíveis aprimoramentos desta operacionalização, considera-se essencial o prosseguimento dos testes de validade do modelo proposto, principalmente no sentido de proceder-se à validação convergente/discriminante à validação nomológica, conforme propõem Adcock y D., 2001.

Desnecessário estender-se acerca das limitações de qualquer modelo e dos perigos de suas operacionalizações, ainda que busquem seguir o caminho metódico do rigor. Sempre há decisões tentativas cujo erro ou acerto apenas será percebido nos próximos testes de validade.

Na medida em que uma operacionalização do modelo de Emerson e outros mature, será possível efetuar aplicações em ampla escala e medir a maturidade colaborativa ao longo do tempo, buscando-se compreender melhor a dinâmica temporal e contextual da governança colaborativa.

Esta iniciativa poderá beneficiar a academia, na medida em que pesquisadores dos meandros da governança colaborativa poderão dispor de um instrumento confiável, e também poderá beneficiar muito o universo de praticantes que lutam todos os dias para fazer seus regimes colaborativos avançar.

\section{Referências}

Adcock, R. y D., C. (2001). Measurement Validity. American Political Science Review, 95(3):529-549.

Agranoff, R. (2007). Managing within networks: Adding value to public organizations. Georgetown University Press.

Agranoff, R. y McGuire, M. (2001). Big questions in pu- 
blic network management research. Journal of public administration research and theory, 11(3):295-326.

Agranoff, R. y McGuire, M. (2004). Collaborative public management: New strategies for local governments. Georgetown University Press.

Agrawal, A. y Lemos, M. C. (2007). A greener revolution in the making?: Environmental governance in the 21st century. Environment: Science and Policy for Sustainable Development, 49(5):36-45.

Ansell, C. y Gash, A. (2008). Collaborative governance in theory and practice. Journal of public administration research and theory, 18(4):543-571.

Axelrod, R. M. (2006). The evolution of cooperation: revised edition. Basic books.

Babbie, E. (2000). The basics of social research. Cengage Learning.

Becker, J., Knackstedt, R., y Pöppelbuß, J. (2009). Developing maturity models for IT management. Business $\&$ Information Systems Engineering, 1(3):213222 .

Bentrup, G. (2001). Evaluation of a collaborative model: a case study analysis of watershed planning in theIntermountain West. Environmental management, 27(5):739-748.

Bititci, U. S., Garengo, P., Ates, A., y Nudurupati, S. S. (2015). Value of maturity models in performance measurement. International journal of production research, 53(10):3062-3085.

Boughzala, I. y De Vreede, G.-J. (2015). Evaluating team collaboration quality: The development and field application of a collaboration maturity model. Journal of Management Information Systems, 32(3):129-157.

Brookes, N. y Clark, R. (2009). Using maturity models to improve project management practice. En POMS 20th Annual Conference, volumen 1.

Bryson, J. M., Crosby, B. C., y Stone, M. M. (2006). The design and implementation of Cross Sector collaborations: Propositions from the literature. Public administration review, 66(s1):44-55.

Chiesa, V., Coughlan, P., y Voss, C. A. (1996). Development of a technical innovation audit. Journal of product innovation management, 13(2):105-136.

CMMI Product Team (2002). Capability Maturity Model Integration (CMMI), Version 1.1 Continuous Representation.

Cooper, T. L., Bryer, T. A., y Meek, J. W. (2006). Citizen-Centered Collaborative Public Management. Public Administration Review, 6(66):76-88.

Costa, R. R. (2016). Governança Colaborativa: Um instrumento de avaliação da maturidade dos regimes de colaboração entre a sociedade e a administração pública. Dissertação de mestrado profissional em administração pública, Escola Brasileira de Administração Pública e de Empresas da Fundação Getulio Vargas.

Costa, S. R. y Ramos, A. F. B. (2013). Modelo de Maturidade em Gerenciamento de Projeto: Um Estudo de Caso Aplicado a Projetos de Petróleo e Energia. Sistemas \& Gestão, 8(3):234-243.

Crosby, B. P. (1979). Quality is free. MacGraw Hill.

Curtis, B., Hefley, B., y Miller, S. (2009). People capability maturity model (P-CMM) version 2.0. Technical report.

Daniels, S. E. y Walker, G. B. (2001). Working through environmental conflict: The collaborative learning approach.

De Bruin, T. y Rosemann, M. (2005). Towards a Business Process Management Maturity Model. En Bartmann, D., Rajola, F., Kallinikos, J., Avison, D., Winter, R., y Ein-Dor, P., editores, Regensburg: ECIS 2005 Proceedings of the Thirteenth European Conference on Information Systems.

Dooley, K., Subra, A., y Anderson, J. (2001). Maturity and its impact on new product development project performance. Research in Engineering Design, 13(1):23-29.

Dourado, L. (2017). COBIT 5: Framework de Governança e Gestão Corporativa de TI.

Emerson, K. y Nabatchi, T. (2015a). Collaborative governance regimes. Georgetown University Press.

Emerson, K. y Nabatchi, T. (2015b). Evaluating the productivity of collaborative governance regimes: A performance matrix. Public Performance $\mathscr{E}$ Management Review, 38(4):717-747.

Emerson, K., Nabatchi, T., y Balogh, S. (2012). An integrative framework for collaborative governance. Journal of public administration research and theory, 22(1):1-29.

Emerson, K., Orr, P. J., Keyes, D. L., y McKnight, K. M. (2009). Environmental conflict resolution: Evaluating performance outcomes and contributing factors. Conflict Resolution Quarterly, 27(1):27-64.

Fischer, R., Ury, W., y Patton, B. (1981). Getting to yes. Negotiating Agreement Without Giving in.

Frank, U. (2004). Referenzmodelle zur ökonomischen Realisierung leistungsfähiger Infrastrukturen für Electronic Commerce. WIRTSCHAFTSINFORMATIK, 46(5):373-381.

Fraser, P., Moultrie, J., y Gregory, M. J. (2002). The use of maturity models/grids as a tool in assessing product development capability. En IEEE International Engineering Management Conference (IEMC 2002): Managing Technology for the New Economy, pp. 244-249, Cambridge. 
Hevner, R., March, S. T., Park, J., y Ram, S. (2004). Design science in information systems research. MIS quarterly, 28(1):75-105.

Innes, J. E. y Booher, D. E. (1999). Consensus building and complex adaptive systems: A framework for evaluating collaborative planning. Journal of the American planning association, 65(4):412-423.

Jucá Jr, A. S. y Amaral, D. (2005). Estudos de caso de maturidade em gestão de projetos em empresas de base tecnológica. En ENCONTRO NACIONAL DE ENGENHARIA DE PRODUÇÃO.

Koppenjan, J. y Klijn, E. (2004). Managing uncertainty in networks: A network approach to problem solving and decision making. Routledge, New York.

Lahrmann, G., Marx, F., Mettler, T., Winter, R., y Wortmann, F. (2011). Inductive design of maturity models: applying the Rasch algorithm for design science research. En International Conference on Design Science Research in Information Systems, pp. 176-191. Springer.

Leach, W. D. (2006). Collaborative public management and democracy: Evidence from western watershed partnerships. Public Administration Review, 66(s1):100-110.

Macbeth, D. K. y Ferguson, N. (1994). Partnership sourcing: An integrated supply chain approach. Financial Times Management/Pitman.

March, S. T. y Smith, G. F. (1995). Design and natural science research on information technology. Decision support systems, 15(4):251-266.

Milward, H. B. y Provan, K. G. (2000). How networks are governed. En Heinrich, C. J. y Lynn, L. E., editores, Governance and performance: New perspectives. Palgrave Macmillan, Washington, D.C.

Moore, M. (1995). Creating Public Value: Strategic Management in government. Harvard University Press, Massachussets.

Moore, M. y Benington, J., editores (2011). Public value: Theory and practice. Palgrave Macmillan, Basingstoke.

Olsen, M. (1965). The logic of collective action: Public goods and the theory of groups.

Ostrom, E. (1990). Governing the commons: The evolution of institutions for collective action. Cambridge University Press, Cambridge.

PM Solutions (2017). What is the Project Management Maturity Model (PMMM)?

Pressman, J. L. y Wildavsky, A. (1973). Implementation. How great expectations in Washington are dashed in Oakland. University of California Press, Berkeley.
Ring, P. S. y Van de Ven, A. H. (1994). Developmental processes of cooperative interorganizational relationships. Academy of management review, 19(1):90118.

Rosa dos Santos, L. (2003). Gestão da maturidade de processos essenciais-convergência para o futuro. RAE-eletrônica, 2(1).

Selin, S. y Chevez, D. (1995). Developing a collaborative model for environmental planning and management. Environmental management, 19(2):189-195.

Siqueira, J. (2017). O Modelo de Maturidade de Processos: como maximizar o retorno dos investimentos em melhoria da qualidade e produtividade.

Spitzer, D. R. (2007). Performance Measurement Maturity. En Transforming Performance Measurement: rethinking the way we measure and drive organizational success, pp. 177-195. AMACOM, New York.

Tarhan, A., Turetken, O., y Reijers, H. A. (2016). Business process maturity models: a systematic literature review. Information and Software Technology, 75:122-134.

Thomson, A. M. y Perry, J. L. (2006). Collaboration processes: Inside the black box. Public administration review, 66(s1):20-32.

Tolbert, P. S. y Zucker, L. G. (1999). A institucionalização da teoria institucional. En Clegg, S., Hardy, C., Nord, W. R., Caldas, M. P., Fachin, R., y Fischer, T., editores, Handbook de Estudos Organizacionais. Atlas, São Paulo.

Wood, D. J. y Gray, B. (1991). Toward a comprehensive theory of collaboration. The Journal of Applied Behavioral Science, 27(2):139-162.

Zelewski, S. (2007). Kann Wissenschaftstheorie behilflich für die Publikationspraxis sein? En Wissenschaftstheoretische Fundierung und wissenschaftliche Orientierung der Wirtschaftsinformatik, pp. 71-120. Berlín. 